\title{
Gust Analysis using Computational Fluid Dynamics Derived Reduced Order Models
}

\author{
S. Timme ${ }^{\mathrm{a}, 1}$, K. J. Badcock ${ }^{\mathrm{a}, 2}$, and A. Da Ronch ${ }^{\mathrm{b}, 3, *}$ \\ ${ }^{a}$ School of Engineering, University of Liverpool, Liverpool L69 3BX, U.K. \\ ${ }^{b}$ Faculty of Engineering and the Environment \\ University of Southampton, Southampton SO17 1BJ, U.K.
}

\begin{abstract}
Time domain gust response analysis based on large order nonlinear aeroelastic models is computationally expensive. An approach to the reduction of nonlinear models for gust response prediction is presented in this paper. The method uses information on the eigenspectrum of the coupled system Jacobian matrix and projects the full order model, through a series expansion, onto a small basis of eigenvectors which is capable of representing the full order model dynamics. The novelty in the paper concerns the representation of the gust term in the reduced model in a manner consistent with standard synthetic gust definitions, allowing a systematic investigation of the influence of a large number of gust shapes without regenerating the reduced model. Results are presented for the Goland wing/store configuration.
\end{abstract}

Keywords: Aeroelasticity, Gust Analysis, CFD, Reduced Order Model, Worst Case Gust, Goland Wing

\footnotetext{
${ }^{*}$ Corresponding author. Email: A.Da-Ronch@soton.ac.uk

${ }^{1}$ Lecturer. Member AIAA.

${ }^{2}$ Professor. Senior member AIAA.

${ }^{3}$ Lecturer. Member AIAA and AIAA Atmospheric Flight Mechanics Technical Committee.
} 


\section{Introduction}

Aircraft regularly encounter atmospheric turbulence, inducing changes in forces and moments, which cause rigid and flexible dynamic responses. These responses introduce loads on the structure which must be accounted for during the design stage to ensure structural integrity. The turbulence is regarded, for linear analysis, as a set of component velocities (gusts) superimposed on the background steady flow. The loads encountered form some of the critical cases used in the structural sizing of a passenger jet. The capability to calculate design loads with a high degree of accuracy would potentially allow reduced conservatism without compromising safety. Currently, conservatism is necessary because of the limited certainty of the possible forms of atmospheric gusts and the limited realism for some flow regimes of linear methods used to predict the aircraft response.

The well-established methods for gust load calculations are based on linear aerodynamic models which are solved in the frequency domain. The use of high-fidelity models based on computational fluid dynamics (CFD) in the research setting has been reported, for example, in Ref. (1). Grid velocities are used to apply a disturbance in a time domain CFD calculation (2), overcoming the problems associated with numerical dissipation of the disturbance but also missing the influence of the aircraft flow field and motion on the gust.

The cost of time domain calculations makes the routine use of CFD in gust response analysis impractical, and system-identification methods have been used as a cheaper alternative. Proper orthogonal decomposition has 
been used as a model reduction technique (3) to generate reduced models for gust simulations, but this method suffers from the usual limitations associated with the necessity for a set of training data closely related to the final application cases, and the difficulty of accounting for nonlinearity in the reduced model. A systematic and cost effective approach to developing reduced models capable of describing both linear and nonlinear effects for a range of cases based on limited development cost has, to date, proved elusive.

An approach to calculating a reduced order model from a large dimension CFD model which can calculate a nonlinear response has been reviewed in Ref. (4). The method first calculates the important modes of the problem from a large order eigenvalue problem. For an aeroelastic limit-cycle oscillation (LCO), the system responds in the critical mode close to the bifurcation point. The approach presented in Refs. (5; 6) is to project the full order model onto the critical mode and expand the residual in a Taylor series, retaining quadratic and cubic terms. The influence of the non-critical space on the critical mode is included through a centre manifold approximation. The method has been successfully applied to various test cases, including the LCO prediction dominated by the motion of a shock wave (5) and a prototype flight dynamics instability of a delta wing (므). The approach to model reduction has been generalized in Ref. (7) by using multiple coupled system eigenmodes for model projection and introducing control deflection and gust interaction effects in the formulation. Reference (8) introduced the flight mechanics degrees of freedom to predict the dynamics of flexible flying aircraft. The method has several strengths, namely: (i) it exploits information from the stability (flutter) calculation for the development of a reduced 
order model (ROM) for dynamic response analyses; (ii) linear or nonlinear reduced models can be developed within the same framework; (iii) the reduced model can be parameterised to avoid ROM regeneration; and (iv) the ROM in state-space form is suitable for control design studies.

The current paper tackles the problem of how to introduce gust terms into the reduced model to allow a gust load analysis to be carried out. The objective is to develop a methodology that allows the reduced model to consider a whole range of gust excitations without recourse to the full order model. The outgrowth of this work is the capability to carry out the search of the worst case gust at no additional costs than those initially encountered in generating the reduced model.

The paper continues with the formulation of the full order aeroelastic model in Sec. 2. The procedure to obtain a reduced model is discussed in Sec. 3. Then a new approach to calculating the gust term in the ROM is proposed. Results are then given in Sec. 4 for a test case to evaluate the method from the point of view of accuracy and computational efficiency. Finally, conclusions are drawn in Sec. 5. The important features of the method developed are: (i) linear and nonlinear ROMs can be derived; and (ii) the model reduction is performed once, with application of any gust made without further recourse to the CFD code.

\section{Full Order Model}

The Euler equations are solved in the curvilinear form on block-structured body-conforming grids: 


$$
\frac{\partial \hat{\mathbf{W}}}{\partial t}+\frac{\partial \hat{\mathbf{F}}}{\partial \xi}+\frac{\partial \hat{\mathbf{G}}}{\partial \eta}+\frac{\partial \hat{\mathbf{H}}}{\partial \zeta}=0
$$

73 The transformation from Cartesian coordinates defines a curvilinear co74 ordinate system from:

$$
\xi=\xi(x, y, z, t), \quad \eta=\eta(x, y, z, t), \quad \zeta=\zeta(x, y, z, t)
$$

75 with the Jacobian determinant of the transformation given by:

$$
J=\left|\frac{\partial(\xi, \eta, \zeta)}{\partial(x, y, z)}\right|
$$

${ }_{76}$ The conserved variables, $\hat{\mathbf{W}}$, and the flux vectors, $\hat{\mathbf{F}}, \hat{\mathbf{G}}$ and $\hat{\mathbf{H}}$, are then 77 defined as follows:

$$
\begin{aligned}
\hat{\mathbf{W}} & =\frac{1}{J} \mathbf{W} \\
\hat{\mathbf{F}} & =\frac{1}{J}\left(\xi_{x} \mathbf{F}+\xi_{y} \mathbf{G}+\xi_{z} \mathbf{H}\right) \\
\hat{\mathbf{G}} & =\frac{1}{J}\left(\eta_{x} \mathbf{F}+\eta_{y} \mathbf{G}+\eta_{z} \mathbf{H}\right) \\
\hat{\mathbf{H}} & =\frac{1}{J}\left(\zeta_{x} \mathbf{F}+\zeta_{y} \mathbf{G}+\zeta_{z} \mathbf{H}\right)
\end{aligned}
$$

78 where the subscripts $\bullet_{x}, \bullet_{y}$ and $\bullet_{z}$ denote differentiation with respect to $x$, 79 $y$ and $z$, respectively. The terms $\mathbf{F}, \mathbf{G}$ and $\mathbf{H}$ are given by: 


$$
\begin{aligned}
& \mathbf{W}=[\rho, \rho u, \rho v, \rho w, \rho E]^{T} \\
& \mathbf{F}=\left[\rho u, \rho u^{2}+p, \rho u v, \rho u w, u(\rho E+p)\right]^{T} \\
& \mathbf{G}=\left[\rho v, \rho u v, \rho v^{2}+p, \rho v w, v(\rho E+p)\right]^{T} \\
& \mathbf{H}=\left[\rho w, \rho u w, \rho v w, \rho w^{2}+p, w(\rho E+p)\right]^{T} .
\end{aligned}
$$

The Euler equations are discretised on curvilinear multiblock body-conforming grids using a cell-centered finite--volume method. The residual is formed using Osher's approximate Riemann solver with the monotone upwind scheme for conservation laws interpolation. Exact Jacobian matrices are formed. The mesh can be deformed using transfinite interpolation. More details on the CFD formulation can be found in Ref. (9), and on the application to problems in aeroelasticity in Ref. (4).

As given in Ref. (4), for general linear structural motions, the dimensionless structural equations of motion are defined in physical coordinates as:

$$
\boldsymbol{M} \delta \ddot{\mathbf{x}}_{s}+\boldsymbol{C} \delta \dot{\mathbf{x}}_{s}+\boldsymbol{K} \delta \mathbf{x}_{s}=\vartheta \mathbf{f}
$$

The deflections $\delta \mathbf{x}_{s}$ of the (linear) structure are defined at the set of physical coordinates $\mathbf{x}_{s}$ by $\delta \mathbf{x}_{s}=\boldsymbol{\Xi} \boldsymbol{\eta}$, where the vector $\boldsymbol{\eta}$ contains the generalised coordinates (modal amplitudes). The columns of the matrix $\boldsymbol{\Xi}$ contain the mode shape vectors evaluated from a finite-element model of the structure with the deflections defined at the structural grid points. Projecting the finite-element equations onto the mode shapes, while scaling to obtain generalised masses of magnitude one (i.e. $\boldsymbol{\Xi}^{T} \boldsymbol{M} \boldsymbol{\Xi}=\boldsymbol{I}$, with $\boldsymbol{I}$ as the identity 
matrix) gives a system of scalar equations written in state--space with the structural residual given by:

$$
\mathbf{R}_{s}=\left[\begin{array}{cc}
\mathbf{0} & \boldsymbol{I} \\
-\boldsymbol{\Xi}^{T} \boldsymbol{K} \boldsymbol{\Xi} & -\boldsymbol{\Xi}^{T} \boldsymbol{C} \boldsymbol{\Xi}
\end{array}\right] \mathbf{w}_{s}+\left[\begin{array}{c}
\mathbf{0} \\
\boldsymbol{I}
\end{array}\right] \vartheta \boldsymbol{\Xi}^{T} \mathbf{f}
$$

and the vector of structural unknowns $\mathbf{w}_{s}=\left[\boldsymbol{\eta}^{T}, \dot{\boldsymbol{\eta}}^{T}\right]^{T}$ containing the generalised coordinates and their velocities. The vector $\mathbf{f}$ of aerodynamic forces (pressure) at the structural grid points follows from the wall pressure, the area of the surface segment and the unit normal vector, and thus is a function of fluid and structural unknowns. It is then projected using the mode shapes to obtain the generalised forces $\boldsymbol{\Xi}^{T} \mathbf{f}$. The parameter $\vartheta$ for the mass ratio is obtained from the nondimensionalisation of the governing equations, and depends on the reference density and the reference length. The method used to transfer the surface pressure forces to the structural nodes is described in Ref. (4).

\subsection{Gust Representation}

Synthetic gusts are defined by space-time functions of a velocity disturbance that propagates through the flow field, interacting with the aircraft. In principle, these disturbances can be introduced through the far field boundary conditions, with the propagation done within the CFD solution. In practice, the gust disturbance will be dissipated by the discretisation. As an alternative, assuming that the gust disturbance propagates without being altered by the background flow field and interaction, a frozen gust can be applied by introducing the gust disturbance through additional contributions to the 
mapping velocity terms $\xi_{t}, \eta_{t}$ and $\zeta_{t}$ in Eqs. (8)-(11). The flow variables are then altered in the discretised version of Eq. (1) through the resulting terms in the fluxes. This approach has been successfully demonstrated for CFD based gust analysis. A schematic in Fig. 1 shows the progressive application of the gust to the grid velocities.

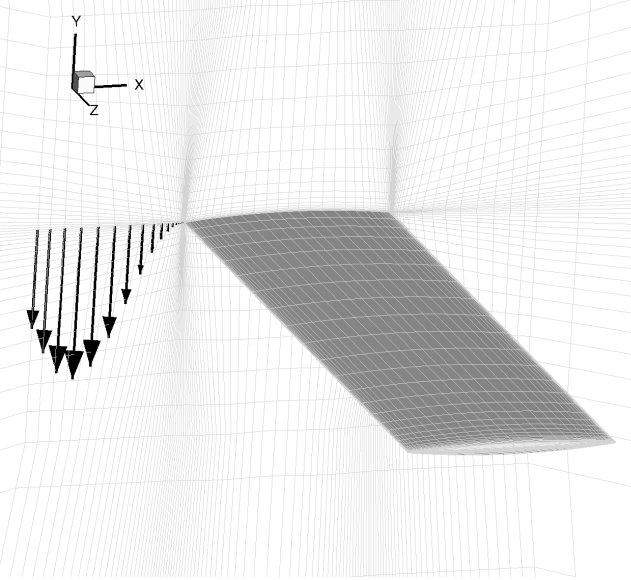

Figure 1: Demonstration of gust application to the CFD; the arrows indicate the grid velocity at each point for a gust of length $6 \mathrm{ft}$; only the points on the symmetry plane for $z=0$ are shown

The disturbances used in this framework are of the discrete and continuous types, see for example Refs. (10; 11). For the vertical component of a discrete gust, for example, the disturbance at each grid point is defined by:

$$
\dot{\boldsymbol{z}}(t)=\boldsymbol{f}(\boldsymbol{x}, t)
$$

where $\dot{\boldsymbol{z}}$ is the vector of the vertical component of the mesh velocities, $\boldsymbol{f}$ is the function defining these velocities, depending on the mesh point location, 
130

131

$\boldsymbol{x}$, and the time instant, $t$. For example, a discrete one-minus-cosine gust, for a single mesh point, is given by:

$$
\dot{z}(t)=\frac{w_{g 0}}{2}\left(1-\cos \left(\frac{2 \pi}{H_{g}}\left(t-t_{0}\right)\right)\right) \quad \text { for } t_{0}<t<t_{0}+H_{g}
$$

where $t_{0}$ is the nondimensional time at which the gust is set to begin, $w_{g 0}$ is the gust intensity, and $H_{g}$ is the nondimensional gust length $\left(H_{g}=L_{g} / c\right.$ where $L_{g}$ is the gust length and $c$ is a characteristic length). In this paper, the gust disturbance applied to each grid point in the mesh is defined as:

$$
\boldsymbol{u}_{d}=[\ldots, \dot{x}, \dot{y}, \dot{z}, \ldots]^{T}
$$

with one triplet of $\dot{x}, \dot{y}$ and $\dot{z}$ for each mesh point.

\section{Model Reduction}

The full order nonlinear aeroelastic model is written in semi-discrete form.

Denote by $\boldsymbol{w}$ the $n$-dimensional state-space vector arising from the fluid and structural spatial discretisation, which is conveniently partitioned into fluid and structural degrees of freedom:

$$
\boldsymbol{w}=\left[\boldsymbol{w}_{f}^{T}, \boldsymbol{w}_{s}^{T}\right]^{T}
$$

The state-space equations in the general vector form are: 


$$
\frac{d \boldsymbol{w}}{d t}=\boldsymbol{R}\left(\boldsymbol{w}, \boldsymbol{u}_{d}\right)
$$

where $\boldsymbol{R}=\left[\boldsymbol{R}_{f}^{T}, \boldsymbol{R}_{s}^{T}\right]$ is the (nonlinear) residual and $\boldsymbol{u}_{d}$ is a vector denoting the applied gust disturbance acting on the system. The homogeneous system has an equilibrium solution, $\boldsymbol{w}_{0}$, for a given constant $\boldsymbol{u}_{d 0}$, corresponding to a constant solution in the state-space and satisfying the aeroelastic equilibrium equation:

$$
\frac{d \boldsymbol{w}_{0}}{d t}=\boldsymbol{R}\left(\boldsymbol{w}_{0}, \mathbf{0}\right)=\mathbf{0}
$$

The system often also includes an independent parameter (freestream speed, air density, altitude, etc.) which is varied to study stability of the equilibria.

Denote by $\Delta \boldsymbol{w}=\boldsymbol{w}-\boldsymbol{w}_{0}$ the increment in the state-space vector with respect to an equilibrium solution (12). The large order nonlinear residual formulated in Eq. (18) is expanded in a Taylor series around the equilibrium point:

$$
\boldsymbol{R}(\boldsymbol{w}) \approx \boldsymbol{A} \Delta \boldsymbol{w}+\frac{\partial \boldsymbol{R}}{\partial \boldsymbol{u}_{d}} \Delta \boldsymbol{u}_{d}+\frac{1}{2} \boldsymbol{B}(\Delta \boldsymbol{w}, \Delta \boldsymbol{w})+\frac{1}{6} \boldsymbol{C}(\Delta \boldsymbol{w}, \Delta \boldsymbol{w}, \Delta \boldsymbol{w})
$$

retaining terms up to third order in the perturbation variable. The treatment of the gust term, which appears as the second term on the right hand side, is considered below. The Jacobian matrix of the coupled system is denoted as $\boldsymbol{A}$, and the vectors $\boldsymbol{B}$ and $\boldsymbol{C}$ indicate, respectively, the second and third order derivative operators. The full order system is projected onto a basis formed by a small number (denoted by $m$ ) of eigenvectors of the Jacobian 
matrix evaluated at the equilibrium position. Right and left eigenvectors are scaled to satisfy the biorthonormality conditions (7). The projection of the full-order model is done using a transformation of coordinates:

$$
\Delta \boldsymbol{w}=\boldsymbol{\Phi} \boldsymbol{z}_{c}+\overline{\boldsymbol{\Phi}} \overline{\boldsymbol{z}}_{c}
$$

where $\boldsymbol{z}_{c} \in \mathbb{C}^{m}$ is the state-space vector governing the dynamics of the reduced order nonlinear system, and $\boldsymbol{\Phi}$ is the matrix of right eigenvectors of $\boldsymbol{A}$. The result is a system of ordinary differential equations in $\boldsymbol{z}_{c}$ which have linear, quadratic and cubic terms in $\boldsymbol{z}_{c}$. The coefficients of these terms are derived by using matrix-free approximations for the first, second and third order derivative operators applied to combinations of the columns of $\boldsymbol{\Phi}$ (i.e. the basis vectors for the reduction). The matrix-free approximations work on residual evaluations, but require extended order arithmetic to be used to obtain accurate approximations. The full details of the methodology are given in Refs. (5; 7; 12; 13).

In the current paper, the linear reduced model, obtained by neglecting the terms $\boldsymbol{B}$ and $\boldsymbol{C}$ in Eq. (20), is generated for gust analysis. Substituting first for $\Delta \boldsymbol{w}$ of Eq. (21) into Eq. (20), and then pre-multiplying by $\overline{\boldsymbol{\Psi}}^{T}$, which is the matrix of left eigenvectors of $\boldsymbol{A}$, one obtains the linear ROM:

$$
\dot{\boldsymbol{z}}_{c}=\operatorname{diag}(\lambda) \boldsymbol{z}_{c}+\overline{\mathbf{\Psi}}^{T} \frac{\partial \boldsymbol{R}}{\partial \boldsymbol{u}_{d}} \Delta \boldsymbol{u}_{d}
$$

where $\operatorname{diag}(\lambda)$ is a diagonal matrix of size $[m, m]$ containing the complex eigenvalues corresponding to the eigenvectors used in the projection. Through manipulation of the terms in $\boldsymbol{B}$ and $\boldsymbol{C}$, a nonlinear ROM can be obtained if required $(7 ; 12)$. 
Finally, it is worth observing that the generation of the ROM is independent from the initial equilibrium point. The coefficients of the ROM, however, depend on the steady-state solution used in the generation process.

\subsection{Nonlinear Eigenvalue Problem}

A major computational challenge arises, when using CFD as the source of the aerodynamic predictions, to calculate the system eigenvectors. To overcome this problem, the Schur complement eigenvalue formulation is used. The coupled system Jacobian matrix of Eq. (18) is most conveniently manipulated by partitioning the matrix as

$$
\boldsymbol{A}=\left[\begin{array}{cc}
\frac{\partial \boldsymbol{R}_{f}}{\partial \boldsymbol{w}_{f}} & \frac{\partial \boldsymbol{R}_{f}}{\partial \boldsymbol{w}_{s}} \\
\frac{\partial \boldsymbol{R}_{s}}{\partial \boldsymbol{w}_{f}} & \frac{\partial \boldsymbol{R}_{s}}{\partial \boldsymbol{w}_{s}}
\end{array}\right]=\left[\begin{array}{cc}
\boldsymbol{A}_{f f} & \boldsymbol{A}_{f s} \\
\boldsymbol{A}_{s f} & \boldsymbol{A}_{s s}
\end{array}\right] .
$$

The block $\boldsymbol{A}_{f f}$ represents the influence of the fluid unknowns on the fluid residual, and has by far the largest number of non-zeros for the structural models used in this paper. The term $\boldsymbol{A}_{f s}$ arises from the dependence of the CFD residual on the mesh motion and speeds, which depend in turn on the structural solution, and is evaluated by finite differences. The term $\boldsymbol{A}_{s f}$ is due to the dependence of the generalized forces on the surface pressures. Finally, the block $\boldsymbol{A}_{s s}$ is the Jacobian of the structural equations with respect to the structural unknowns.

Write the coupled system eigenvalue problem as:

$$
\left[\begin{array}{cc}
\boldsymbol{A}_{f f} & \boldsymbol{A}_{f s} \\
\boldsymbol{A}_{s f} & \boldsymbol{A}_{s s}
\end{array}\right] \boldsymbol{\phi}=\lambda \boldsymbol{\phi}
$$


where $\phi$ and $\lambda$ are the complex eigenvector and eigenvalue, respectively. Partition the eigenvector as:

$$
\phi=\left[\phi_{f}^{T}, \phi_{s}^{T}\right]^{T}
$$

In Eq. (24), substituting $\phi_{f}$ from the first set of equations into the second set of equations, one finds that the eigenvalue $\lambda$, assuming it is not an eigenvalue of $\boldsymbol{A}_{f f}$, satisfies the nonlinear eigenvalue problem:

$$
\boldsymbol{S}(\lambda) \boldsymbol{\phi}_{s}=\lambda \boldsymbol{\phi}_{s}
$$

where $\boldsymbol{S}(\lambda)=\boldsymbol{A}_{s s}-\boldsymbol{A}_{s f}\left(\boldsymbol{A}_{f f}-\lambda I\right)^{-1} \boldsymbol{A}_{f s}$. The matrix $\boldsymbol{S}(\lambda)$ is the sum of the structural matrix and a second term arising from the coupling of the fluid and structure. Equation (26), which is a nonlinear eigenvalue problem, is solved using Newton's method. To overcome the cost of forming the residual and its Jacobian matrix at each iteration, an approximation of $\left(\boldsymbol{A}_{f f}-\lambda I\right)^{-1}$ is used. The calculation of the left eigenvector $\boldsymbol{\psi}$ involves solving the adjoint problem of Eq. (24). More details on the Schur complement eigenvalue solver and its application to realistically sized aeroelastic models can be found in Ref. (14).

\subsection{Gust Term in the Reduced Order Model Setting}

As described above, the gust is introduced into the full order model through the grid velocities, represented in Eq. (18) by the vector $\boldsymbol{u}_{d}$. The treatment of this component in the reduced model is the main contribution of this paper. The challenge is to manipulate the term $\frac{\partial \boldsymbol{R}}{\partial \boldsymbol{u}_{d}} \Delta \boldsymbol{u}_{d}$ in Eq. 22 
so that it is represented in a convenient way in the reduced model. Using the chain rule, the dependence of the nonlinear full order residual on the gust perturbation is rewritten as:

$$
\frac{\partial \boldsymbol{R}}{\partial \boldsymbol{u}_{d}}=\frac{\partial \boldsymbol{R}}{\partial \boldsymbol{u}} \frac{\partial \boldsymbol{u}}{\partial \boldsymbol{u}_{d}}
$$

where $\boldsymbol{u}$ is a vector of mesh velocities. The first term on the right side depends on mesh point velocities only and can be computed independently of the gust definition using finite differences, analytical or automatic differentiation.

The second term on the right side of Eq. (27) depends on both spatial and temporal coordinates. The reason for this is that, recalling Eq. (14), the prescribed gust is in general a function of space and time. The gust simulation using a ROM, as formulated in Refs. (7; 10), requires at each time step the calculation of the contribution arising from

$$
\bar{\psi}^{T} \frac{\partial \boldsymbol{R}}{\partial \boldsymbol{u}} \frac{\partial \boldsymbol{u}}{\partial \boldsymbol{u}_{d}} \Delta \boldsymbol{u}_{d}
$$

The first two terms involve a matrix-matrix multiplication, and this can be carried out once during the generation of the ROM calculation independently of the gust definition. This defines a matrix, $\gamma$, which is constant and independent of the gust shape. The term $\frac{\partial \boldsymbol{u}}{\partial \boldsymbol{u}_{d}}$ is simply the identity matrix when using the field velocity method to prescribe the gust, and $\boldsymbol{u}_{d}$ is the time varying vector defining the propagation in time and space of the gust disturbances. At each time step iteration for solving the ROM, the vector on the right side needs to be updated to account for the gust translation, and a matrix-vector multiplication is then needed. It is worth noting that the 
CFD code does not need to be accessed for this operation, which requires only the grid point coordinates, and the ROM can be applied to any gust shape (discrete and continuous).

The linear reduced model is then written as:

$$
\dot{\boldsymbol{z}}_{c}=\operatorname{diag}(\lambda) \boldsymbol{z}_{c}+\boldsymbol{\gamma}^{T} \Delta \boldsymbol{u}_{d}
$$

Before proceeding to analyse the computational cost and general predictive capabilities of the reduced model, considerations are given about the underlying assumptions. First, the linear ROM is as accurate as the nonlinear coupled solver in the limiting case that the response is small around the reference equilibrium. With second order effects dominant, that are characterised, for example, by strong moving shocks and large structural deformations, the predictions will degrade. Second, the model projection relies on a dominant subspace of coupled modeshapes that reproduce the relevant dynamics of the full model. If needed, the basis for projection may be enriched by selection of additional modeshapes. The last consideration is about the Schur complement eigenvalue problem. This approach overcomes the limitation of the standard $\mathrm{p}-\mathrm{k}$ method, which is valid for undamped vibrations, because it provides a correct identification of the aeroelastic damping using linearised CFD aerodynamics.

\section{Results}

For conciseness, the test case is for the Goland wing. Other test cases may be found in the references herein provided. In particular, the interested 
reader is referred to Ref. (7) for the initial investigation on a wing typical section, Ref. (10) regarding a three-dimensional wing test case, and Ref. (11) for the extension to a passenger transport aircraft.

The Goland wing has a chord of $6 \mathrm{ft}$ and a span of $20 \mathrm{ft}$. It is a rectangular cantilevered wing with a $4 \%$ thick parabolic section. The structural model for the wing/store configuration follows the description given in Ref. (15). The four mode shapes shown in Fig. 2 were retained for the aeroelastic simulations herein presented. The CFD grid for Euler simulations has about 400,000 points. All simulations are done for a freestream Mach number of 0.85 and one degree angle of attack chosen to allow the influence of static deformation on the symmetric wing model.

First, a stability calculation was made using the Schur complement method as in Ref. (16). The traces of the aeroelastic eigenvalues are shown in Fig. 3 as a function of the equivalent airspeed (EAS). One thousand altitude steps for the altitude traces were employed. The wing model shows the typical bending-torsion type of instability. The eigenvectors for the model reduction were computed at the subcritical altitude of $40,000 \mathrm{ft}$ corresponding to $408 \mathrm{ft} / \mathrm{s}$ EAS.

Then, the ROM was calculated with the gust terms. Four aeroelastic modes, corresponding to the four structural normal modes in Figure 2, were used for the reduction. The coefficients of the linear reduced model, without reporting the gust term, were found to be:

$$
\dot{\boldsymbol{z}}_{c}=\operatorname{diag}\left(\lambda_{1}, \lambda_{2}, \lambda_{3}, \lambda_{4}\right) \boldsymbol{z}_{c}
$$

where $\lambda_{1}=-1.636 \cdot 10^{-3}+7.888 \cdot 10^{-2} i, \lambda_{2}=-9.453 \cdot 10^{-3}+1.209 \cdot 10^{-1} i$, 

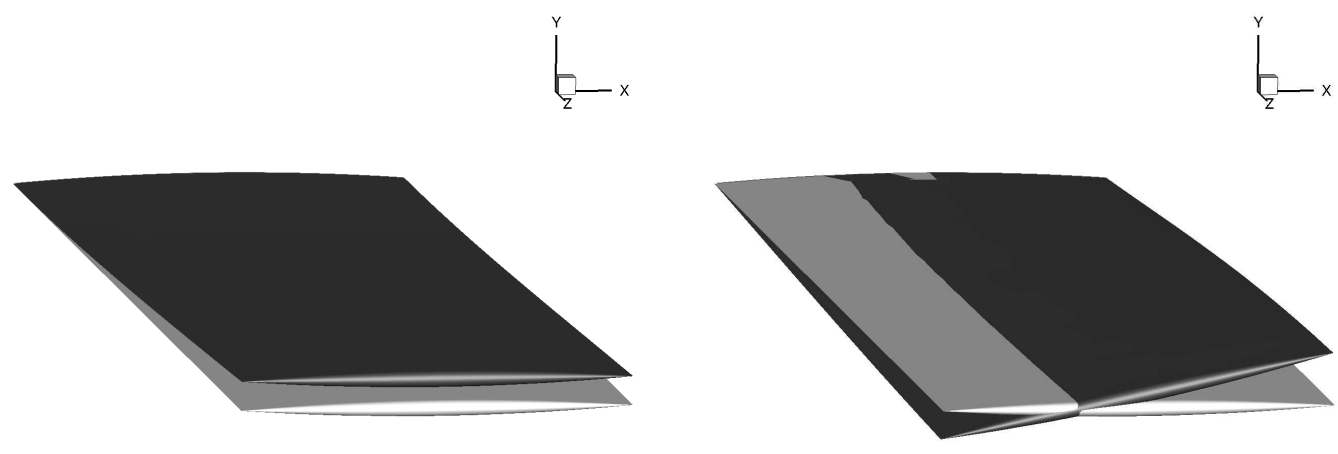

(a) Mode $1(1.72 \mathrm{~Hz})$

(b) Mode $2(3.05 \mathrm{~Hz})$
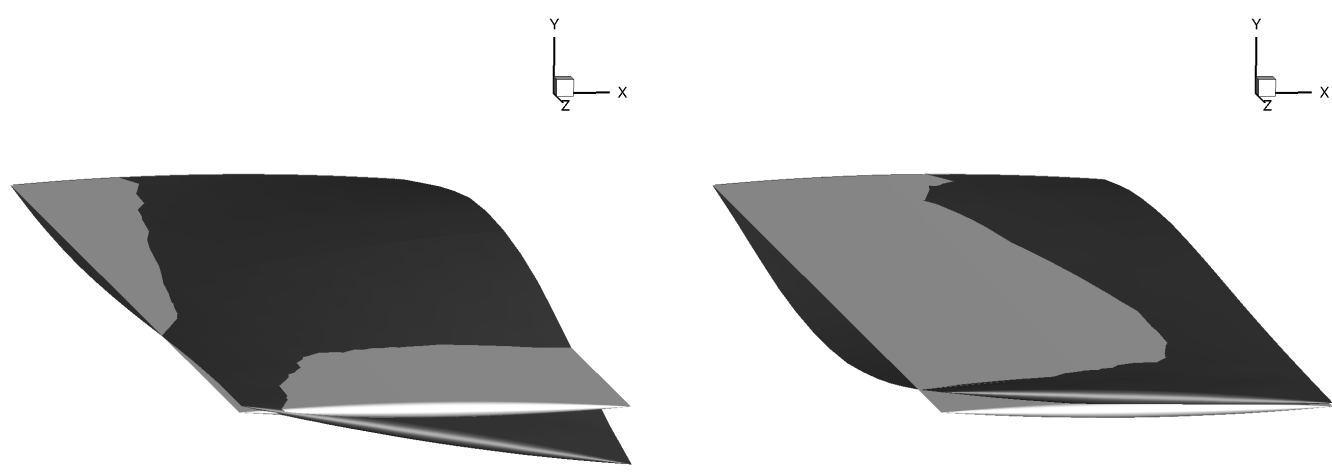

(c) Mode $3(9.18 \mathrm{~Hz})$

(d) Mode $4(11.10 \mathrm{~Hz})$

Figure 2: Modeshapes for the Goland wing/store configuration; for illustration purposes, a modal amplitude of 4 is used 


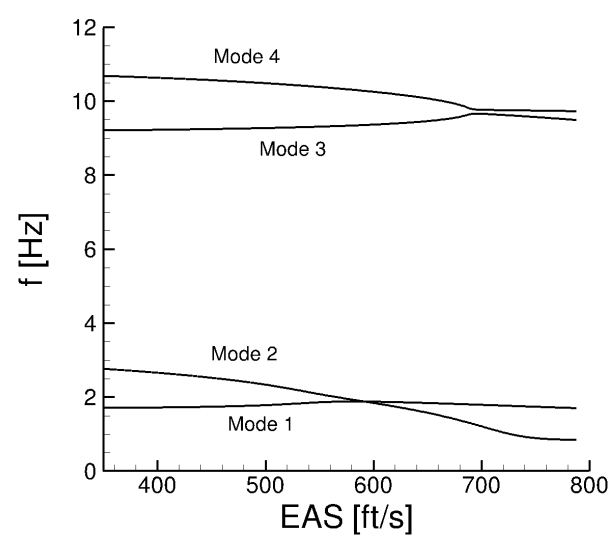

(a) Damped natural frequency, $f$

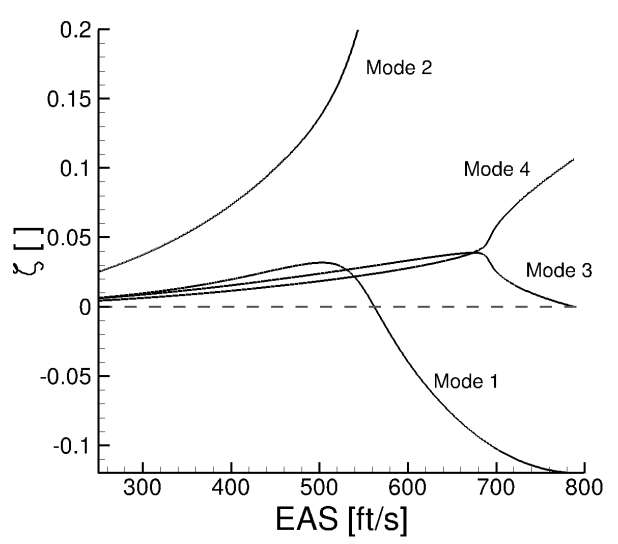

(b) Damping ratio, $\zeta$

Figure 3: Eigenvalue traces for Goland wing/store configuration (Mach 0.85, one degree angle of attack)

$\lambda_{3}=-5.027 \cdot 10^{-3}+4.229 \cdot 10^{-1} i$, and $\lambda_{4}=-7.716 \cdot 10^{-3}+4.867 \cdot 10^{-1} i$.

Table 1 compares the computational efficiency of the reduced model against that of the full order model. All calculations, based on full and reduced models, were run on a single process of a 4 -core Intel Xeon $3.3 \mathrm{GHz}$ computer, and a nondimensional time step of 0.01 was used. For comparison, computational costs were normalised by the cost of the time domain full order model. It is worth noting that smaller time steps would likely be required for viscous simulations, with longer time histories also needed to determine a response involving a wider range of frequencies. The reduced model generation times do not scale with these factors, and hence the timings given in Table 1 are considered conservative. A recent application to a viscous simulation is reported in Ref. (17). Timings start from a precursor eigenvalue calculation which would be done as part of a flutter calculation. 
The generation of the ROM, which consists of the eigenvector calculation and the calculation of the gust term, $\gamma$, takes about $13 \%$ of the cost of the full order time response calculation. The time integration of the reduced model, Eq. $(29)$, is essentially free.

\begin{tabular}{lc}
\hline \hline \multicolumn{1}{c}{ Step } & Cost \\
\hline Time Domain Full Order Calculation & $1 \cdot 10^{0}$ \\
\hline Reduced Model Generation: & \\
a) Calculating Eigenvector Basis & $3 \cdot 10^{-2}$ \\
b) Calculating Gust Vector, $\gamma$ & $1 \cdot 10^{-1}$ \\
Time Domain Reduced Model Calculation & $1 \cdot 10^{-5}$ \\
\hline \hline
\end{tabular}

Table 1: Computational cost for the generation and use of the ROM for gust analysis

To illustrate the potential benefits of the reduced model, the worst case gust search was carried out for the one-minus-cosine family of gusts. The wing response is characterised by the displacement at the wing tip leading and trailing edges, and the resulting twist of the wing tip. Figure 4 shows the peaks of the response for different gust lengths computed by the full order (CFD) and reduced (ROM) models. The reduced model was generated once, and then deployed for the worst case gust search at no additional costs. A good agreement, for the purpose of rapid engineering simulations, between the reduced and full order predictions was found. The worst case gust is for a gust length of approximately $400 \mathrm{ft}$ at a speed of $408 \mathrm{ft} / \mathrm{s}$ EAS, which excites the response predominantly in the first bending mode (normal mode at $1.72 \mathrm{~Hz}$ ). The time responses for different gust lengths are shown in Fig. 5 , 
and confirm the predictive general capabilities of the reduced model for gust response analysis.

\section{Conclusions}

The introduction of a gust into a reduced model in a manner consistent with well-established gust definitions has been considered. A new method was proposed that allows a one-off model reduction, with any gust subsequently applied to the reduced model. The formulation allows linear or nonlinear reduced models to be derived, based on a range of full order modelling options, including linear or nonlinear structural models, and linear or CFD aerodynamic models. In the current paper, linear reduced models of the CFD have proved adequate for the gust interaction simulations. Results were presented for a wing test case (Golang wing/store configuration) to demonstrate the capability of the method. The ability of the method to enable calculations for a variety of gusts was illustrated.

\section{Acknowledgments}

This work was supported by the U.K. Engineering and Physical Sciences Research Council (EPSRC) under grant EP/I014594/1.

\section{References}

[1] D. E. Raveh, Gust-response analysis of free elastic aircraft in the transonic flight regime, Journal of Aircraft 48 (4) (2011) 1204-1211. doi:10.2514/1.C031224. 


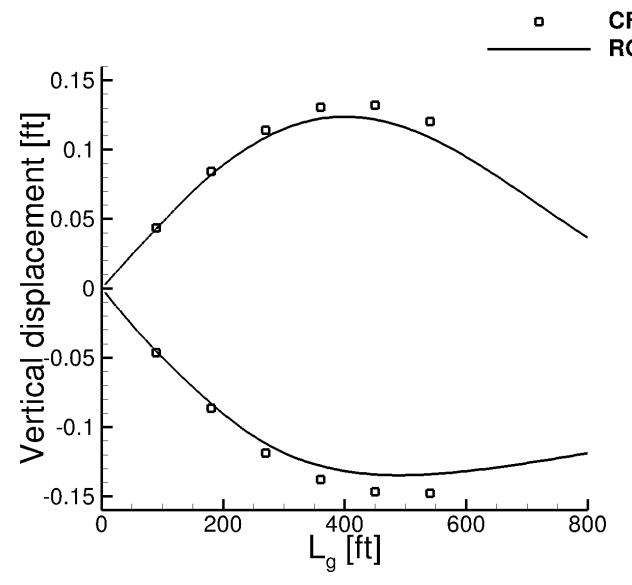

(a) Wing tip leading edge

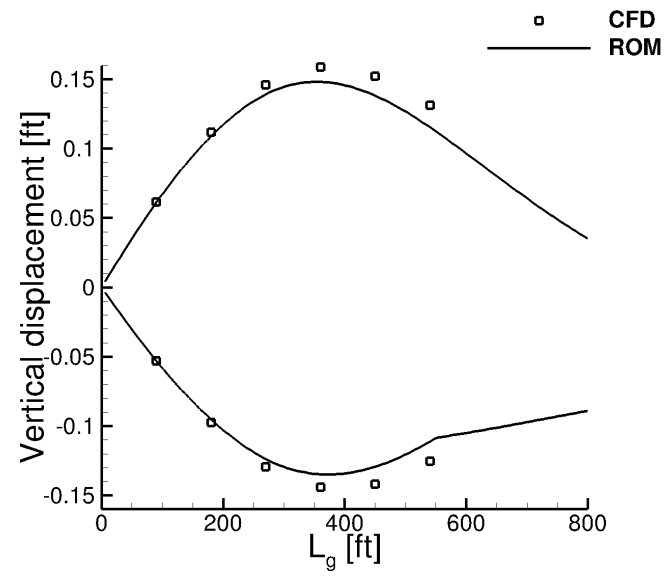

(b) Wing tip trailing edge

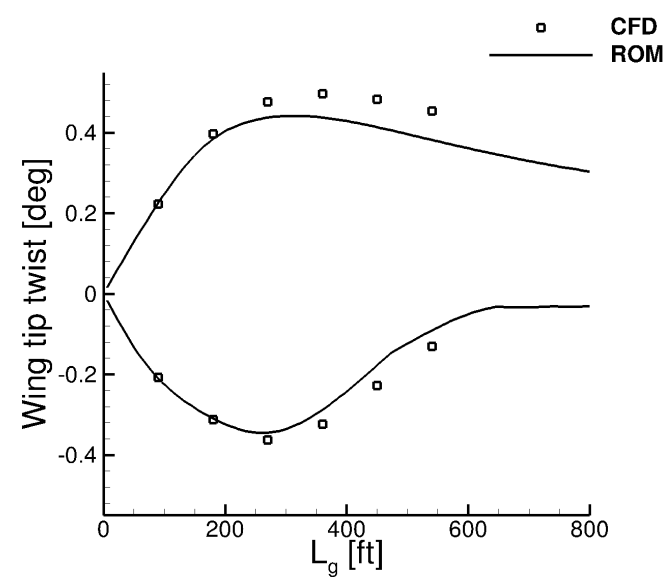

(c) Wing tip twist

Figure 4: Worst case gust search for the Goland wing/store configuration for a one-minuscosine gust family (gust intensity $1 \%$ of the freestream velocity, Mach 0.85 , and altitude $40,000 \mathrm{ft}$ ) 


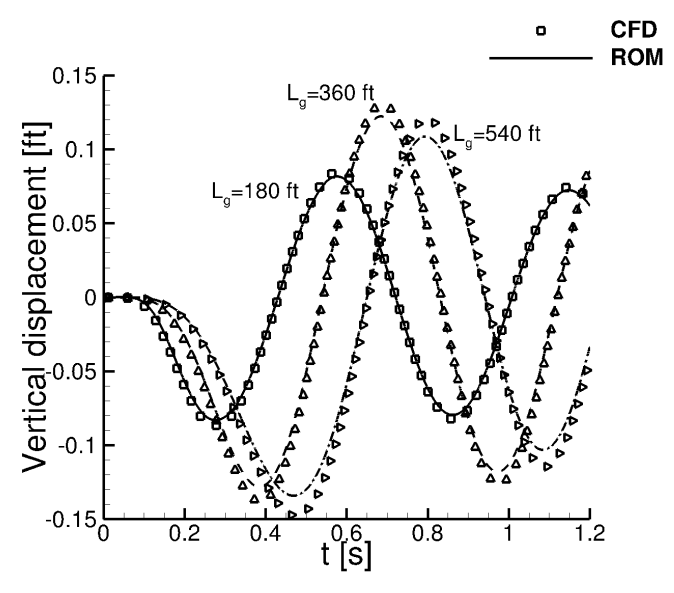

(a) Wing tip leading edge

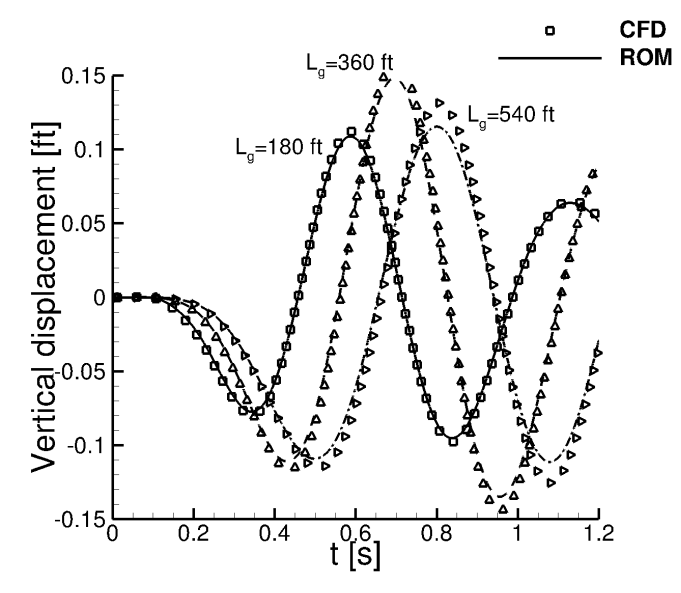

(b) Wing tip trailing edge

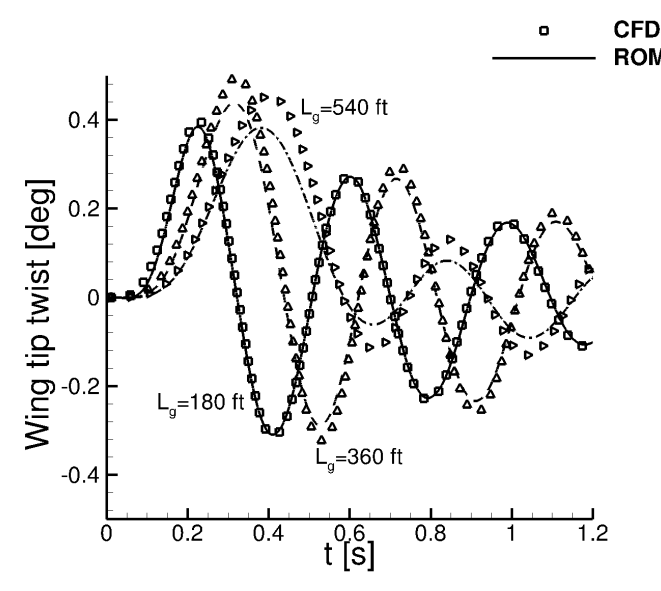

(c) Wing tip twist

Figure 5: Gust responses to a one-minus-cosine gust for gust lengths of 180, 360 and 540 $\mathrm{ft}$ (gust intensity $1 \%$ of the freestream velocity, Mach 0.85 , and altitude 40,000 ft) 
[2] V. Parameswaran, J. D. Baeder, Indicial aerodynamics in compressible flow - direct computational fluid dynamic calculations, Journal of Aircraft 34 (1) (1997) 131-133. doi:10.2514/2.2146.

[3] Q. Zhou, D. Li, A. D. Ronch, G. Chen, Y. Li, Computational fluid dynamics-based transonic flutter suppression with control delay, Journal of Fluids and Structures 66 (2016) 183-206. doi:10.1016/j. jfluidstructs.2016.07.002.

[4] K. J. Badcock, S. Timme, S. Marques, H. Khodaparast, M. Prandina, J. E. Mottershead, A. Swift, A. D. Ronch, M. A. Woodgate, Transonic aeroelastic simulation for instability searches and uncertainty analysis, Progress in Aerospace Sciences 47 (5) (2011) 392-423. doi:10.1016/j . paerosci.2011.05.002.

[5] M. A. Woodgate, K. J. Badcock, Fast prediction of transonic aeroelastic stability and limit cycles, AIAA Journal 45 (6) (2007) 1370-1381. doi: $10.2514 / 1.25604$.

[6] K. J. Badcock, M. A. Woodgate, M. R. Allan, P. S. Beran, Wing-rock limit cycle oscillation prediction based on computational fluid dynamics, Journal of Aircraft 45 (3) (2008) 954-961. doi:10.2514/1.32812.

[7] A. Da Ronch, K. J. Badcock, Y. Wang, A. Wynn, R. N. Palacios, Nonlinear model reduction for flexible aircraft control design, in: AIAA Atmospheric Flight Mechanics Conference, AIAA Paper 2012-4404, Minneapolis, MN, 2012. doi:10.2514/6.2012-4404. 
[8] N. D. Tantaroudas, A. Da Ronch, K. J. Badcock, R. Palacios, Model order reduction for control design of flexible free-flying aircraft, in: AIAA Science and Technology Forum and Exposition, AIAA Paper 2015-0240, Kissimmee, FL, 2015. doi:10.2514/6.2015-0240.

[9] K. J. Badcock, B. E. Richards, M. A. Woodgate, Elements of computational fluid dynamics on block structured grids using implicit solvers, Progress in Aerospace Sciences 36 (5-6) (2000) 351-392. doi:10.1016/ S0376-0421(00) 00005-1.

[10] A. Da Ronch, N. D. Tantaroudas, S. Timme, K. J. Badcock, Model reduction for linear and nonlinear gust loads analysis, in: 54th AIAA/ASME/ASCE/AHS/ASC Structures, Structural Dynamics, and Materials Conference, AIAA Paper 2013-1492, Boston, MA, 2013. doi:10.2514/6.2013-1492.

[11] S. Timme, K. J. Badcock, A. Da Ronch, Linear reduced order modelling for gust response analysis using the dlr-tau code, in: International Forum on Aeroelasticity and Structural Dynamics (IFASD), IFASD Paper 2013-36A, Bristol, U.K., 2013.

[12] N. D. Tantaroudas, A. Da Ronch, Nonlinear reduced order aeroservoelastic analysis of very flexible aircraft, in: P. Marqués, A. Da Ronch (Eds.), Novel Concepts in Unmanned Aircraft Aerodynamics, Flight Stability, and Control, Wiley-Blackwell, Chichester, Great Britain, 2016, ESBN10: 1118928687, ISBN-13: 978-1118928684.

[13] K. J. Badcock, H. H. Khodaparast, S. Timme, J. E. Mottershead, Cal- 
culating the influence of structural uncertainty on aeroelastic limit cycle response, in: 52nd AIAA/ASME/ASCE/AHS/ASC Structures, Structural Dynamics, and Materials Conference, AIAA Paper 2011-1741, Denver, CO, 2011. doi:10.2514/6.2011-1741.

[14] K. J. Badcock, M. A. Woodgate, Bifurcation prediction of large-order aeroelastic models, AIAA Journal 48 (6) (2010) 1037-1046. doi:10. $2514 / 1.40961$.

[15] P. S. Beran, N. S. Khot, F. E. Eastep, R. D. Snyder, J. V. Zweber, Numerical analysis of store-induced limit-cycle oscillation, Journal of Aircraft 41 (6) (2004) 1315-1326. doi:10.2514/1.404.

[16] S. Timme, S. Marques, K. J. Badcock, Transonic aeroelastic stability analysis using a kriging-based Schur complement formulation, AIAA Journal 49 (6) (2011) 1202-1213. doi:10.2514/1.J050975.

[17] P. Bekemeyer, S. Timme, Reduced order gust response simulation using computational fluid dynamics, in: 57th AIAA/ASCE/AHS/ASC Structures, Structural Dynamics, and Materials Conference, AIAA SciTech Forum, AIAA Paper 2016-1485, San Diego, CA, 2016. doi:10.2514/ $6.2016-1485$. 